\title{
STUDY OF THE EFFECT OF MIDAZOLAM PRIOR TO ELECTIVE CESAREAN SECTION ON MOTHER AND NEONATE UNDER SPINAL ANESTHESIA
}

\author{
By
}

\section{Emad Abd El-Hamid Shaaban, Mohamed Abd El-Gawad Abd El-Halim Abu El-Seoud and Mahmoud Emad El-Din Sabra Abd El-Ghani *}

Anesthesiology and Intensive Care Department, Faculty of Medicine, Al-Azhar University

* Corresponding author: Mahmoud Emad Eldin Sabra Abdel Ghani, E-mail: ma7moud.sabra@gmail.com

\begin{abstract}
Background: Spinal anesthesia is the most popular form of regional anesthesia used for Cesarean delivery. Like all surgical patients, obstetric patients also feel operative stress and anxiety. The pharmacological sedation in spinal anesthesia is not commonly applied.
\end{abstract}

Objectives: This work aimed to determine the effect of premedication with midazolam on preoperative maternal anxiety, neonatal outcome regarding respiratory depressant effect and muscle tone, and also postoperative satisfaction of parturients scheduled for elective cesarean delivery under spinal anesthesia.

Patients and Methods: After approval of Institutional ethical committee and obtaining written informed consent from eligible parturient women, 80 full term pregnant patients aged between 18 and 40 years were classified according to American Society of Anesthesiologist I and II and scheduled for elective cesarean section. The study was conducted in the Obstetrics Department of Al-Azhar University Hospitals (AlHussein and Bab-Al-Shaarya), from December 2018 till June 2019 .

Results: Midazolam intravenously at a dose of $0.035 \mathrm{mg} / \mathrm{kg}$ before spinal anesthesia for patients undergoing cesarean section were effective in relieving maternal anxiety with no adverse effect on neonates or maternal hemodynamic instability.

Conclusion: Preoperative sedation 30 mins pre anesthetichally was found to be associated with improved preoperative anxiety, postoperative satisfaction, and maternal hemodynamics in parturients scheduled for elective cesarean delivery.

Keywords: Midazolam, Cesarean Section, Neonate

\section{INTRODUCTION}

The worldwide number of cesarean sections has increased (Martin et al., 2010), and the safety of the mother and child is important to select the method of anesthesia and its administration carefully (Zhou et al., 2018).

Spinal anesthesia used for Cesarean delivery due to its benefits of simplicity, low airway complications, facilitation for postoperative analgesia, decreased blood loss, less neonatal exposure to potentially depressant drugs, and conscious mother at the birth time that creates effective maternal-infant bonding (Sakata et al., 2017).

Stress intensity is influenced by numerous factors such as previous pain experiences, education, culture, expectations, environmental factors and 
support from care givers (Carroll et al., 2012). Some drawbacks are linked with regional anesthesia techniques: pain at the puncture site, fear of needles and recall of the procedure (Patki and Shelgaonkar, 2011).

Stress response leads to release of catecholamines and other vasopressors. At full term, uterine vasculature is maximally dilated, but still responds to these vasopressors causing uterine vasoconstriction and decrease the uterine and placental blood flow which adversely affect the neonates (Amin et al., 2011). Therefore, the prevention from maternal stress is potentially important. This can be prevented by giving patients detailed information about their operation and with preoperative pharmacological medications (Senel and Morgan, 2014).

Midazolam is a highly efficient preoperative sedative and anxiolytic with antegrade amnesia properties (Griffin et al., 2013).

This work was aiming to determine the effect of premedication with midazolam on preoperative maternal anxiety, neonatal outcome regarding respiratory depressant effect and muscle tone and also postoperative satisfaction of parturients scheduled for elective cesarean delivery under spinal anesthesia.

\section{PATIENTS AND METHODS}

After approval of Institutional ethical committee and obtaining written informed consent from eligible parturient women, 80 full term pregnant patients aged between 18 and 40 years, classified according to American Society of Anesthesiologist (ASA) I and II, scheduled for elective cesarean section have been enrolled in the current placebocontrolled prospective randomized study. The study was conducted in the Obstetrics Department of Al-Azhar University Hospitals "Al-Hussein and Bab-Al-Shaarya", Cairo, Egypt, from December 2018 till June 2019.

All patients were divided into two equal groups, normal saline control group (Group A) and midazolam group (Group B), based on the predetermined method of randomization using a computergenerated randomization list and a sealed envelope technique.

Group A, received placebo of $3 \mathrm{ml}$ of normal saline in the preoperative waiting room, 30 minutes before surgery.

Group B, received $0.035 \mathrm{mg} / \mathrm{kg}$ IV Midazolam in the preoperative waiting room, 30 minutes before surgery diluted in $3 \mathrm{ml}$.

The study solution (normal saline or midazolam) was prepared in a $3 \mathrm{~mL}$ volume, by an anesthesiologist not involved in the care of the woman or collecting the data for the study. Another anesthesiologist who was blinded to the study provided perioperative care and collected perioperative data.

All patients were booked on a morning list, instructed for the same preoperative fasting protocol and were approved, and educated as to how to complete the questionnaire required for the study.

\section{Exclusion criteria:}

Patients with the following criteria were excluded from the study:

1. Non-elective cases.

2. Multiple pregnancies and preterm pregnancies. 
3. Known fetal anomalies and retarded fetal growth ( $\leq 2500 \mathrm{gm}$.).

4. Pathologies that might affect the acidalkaline balance.

5. Patients with diabetes mellitus or hypertension.

6. Meconium/amniotic fluid aspiration.

7. Cases contraindicated for regional anesthesia.

\section{Preoperative assessment included:}

1. Full history taking including coexisting comorbidities or complications such as diabetes mellitus, hypertension, dyslipidemia, history of cerebrovascular stroke ...etc.

2. Clinical examination.

3. Routine laboratory investigations including complete blood count (CBC), random blood sugar (RBS), liver function test (LFT), kidney function test (KFT), prothrombin time (PT) and activated partial thromboplastin time (a PTT).

4. All the patients' basic demographic information were recorded.

\section{Maternal Data and Measurements:}

- Demographic characteristics: Age (in years), height (in $\mathrm{cm}$ ), weight (in $\mathrm{Kg}$ ) and Body mass index $(\mathrm{kg} / \mathrm{m} 2)$.

- Spinal to delivery time (minutes).

- Hemodynamic parameters (heart rate, mean arterial blood pressure, and saturation using pulse oximetry), all are recorded at baseline, just after injection and for two hours post-operative. The data obtained at baseline; on arrival to the preoperative waiting room (T0), 5 min after Midazolam/Placebo injection
(T1), 5 min after spinal anesthesia (T2); then parameters were respectively recorded every 10 minutes up to two hours (T3-T14).

- Total O2 flow increments requirement (L/min) to keep $\mathrm{SpO} 2 \geq 95 \%$.

- Patient anxiety was evaluated according to the Amsterdam Preoperative Anxiety and Information Scale (APAIS). It is a questionnaire that consists of six questions receiving a score of 30 , investigating patients' concerns and anxieties (Goebel et al., 2011).

- APAIS was applied blindly two times: Firstly, in a preoperative visit before transfer to the operating theater, and secondly, $5 \mathrm{~min}$ later after administration of midazolam.

After collecting all data blindly, data of corresponding events were compared, i.e. data in relation to study drug administration and spinal anesthesia time.

Neonatal Data and Measurements: Newborns were evaluated using Apgar score (Kemper et al., 2011), neurologic and Adaptive Capacity Score (NACS) (Hashemi et al., 2015), and umbilical artery blood gases.

\section{Ending point of study:}

- Patient refusal to continue the study at any time.

- Two hours from surgery after fulfilling discharge criteria.

- Induction of general anesthesia.

- Presence of one of the exclusion criteria. 
Statistical Analysis:

Data were analyzed using Statistical Package for Social Sciences, version 20.0 (SPSS Inc., Chicago, Illinois, USA). Quantitative data were expressed as mean \pm standard deviation (SD). Qualitative data were expressed as frequency and percentage. Independent-samples t-test of significance was used when comparing between two means. Chi-square (X2) test of significance was used in order to compare proportions between two qualitative parameters. P-value $<0.05$ was considered significant.

\section{RESULTS}

There were no significant differences regarding demographic characteristics (age, body weight, height and ASA score Table 1).

Table (1): Comparison between control group and midazolam group according to demographic data of mothers

\begin{tabular}{|c|c|c|c|}
\hline $\begin{array}{ll}\text { Demographic Data } & \text { Groups } \\
\end{array}$ & $\begin{array}{c}\text { Control group } \\
(n=40)\end{array}$ & $\begin{array}{l}\text { Midazolam group } \\
(n=40)\end{array}$ & p-value \\
\hline $\begin{array}{l}\text { Age (years) } \\
\text { Mean } \pm \text { SD } \\
\text { Range }\end{array}$ & $\begin{array}{c}26.98 \pm 4.89 \\
21-37\end{array}$ & $\begin{array}{c}28.55 \pm 4.68 \\
21-38\end{array}$ & $>0.05$ \\
\hline $\begin{array}{l}\text { Weight (kg) } \\
\text { Mean } \pm \text { SD } \\
\text { Range }\end{array}$ & $\begin{array}{c}69.58 \pm 6.11 \\
60-80\end{array}$ & $\begin{array}{c}68.95 \pm 6.62 \\
60-86\end{array}$ & $>0.05$ \\
\hline $\begin{array}{l}\text { Height (cm) } \\
\text { Mean } \pm \text { SD } \\
\text { Range }\end{array}$ & $\begin{array}{c}159.03 \pm 2.73 \\
155-165\end{array}$ & $\begin{array}{c}159.95 \pm 4.08 \\
150-165\end{array}$ & $>0.05$ \\
\hline $\begin{array}{l}\text { ASA } \\
\text { I } \\
\text { II }\end{array}$ & $\begin{array}{c}31(77.5 \%) \\
9(22.5 \%)\end{array}$ & $\begin{array}{l}30(75 \%) \\
10(25 \%)\end{array}$ & $>0.05$ \\
\hline
\end{tabular}

Amsterdam Preoperative Anxiety and Information Scale (APAIS) showed significant difference between control group and midazolam group At (A2) 5 mins after administration of midazolam (Table 2).

Table (2): Comparison between control group and midazolam group according to Amsterdam preoperative anxiety and information scale of mothers

\begin{tabular}{|l|c|c|c|}
\hline $\begin{array}{l}\text { Amsterdam } \\
\text { Preoperative Anxiety and } \\
\text { Information Scale (APIAS) }\end{array}$ & $\begin{array}{c}\text { Control } \\
\text { group } \\
(\mathbf{n = 4 0})\end{array}$ & $\begin{array}{c}\text { Midazolam } \\
\text { group } \\
(\mathbf{n = 4 0})\end{array}$ & p-value \\
\hline $\begin{array}{l}\mathbf{1} \mathbf{2} \mathbf{h} \text { before their transfer to the operating theater } \\
\mathbf{( A 1 )}\end{array}$ & $19.60 \pm 2.11$ & $19.43 \pm 1.72$ & $>0.05$ \\
Mean \pm SD & $16-23$ & $17-22$ & \\
Range & & & \\
\hline $\mathbf{5}$ mins After Adminstration of midazolam & $17.33 \pm 1.80$ & $13.83 \pm 1.47$ & $<0.001 * *$ \\
$\mathbf{( A 2 )}$ & $14-20$ & $12-16$ & \\
Mean \pm SD & & \\
Range & & \\
\hline
\end{tabular}


Heart rate values showed statistically from T1 to T3 (Table 3). significant differences between the groups

Table (3): Comparison between control group and midazolam group according to heart rate of mothers

\begin{tabular}{|c|c|c|c|}
\hline $\begin{array}{ll}\text { Heart Rate (beat/min) } & \text { Groups } \\
\end{array}$ & $\begin{array}{c}\text { Control group } \\
(n=40)\end{array}$ & $\begin{array}{c}\text { Midazolam group } \\
(n=40)\end{array}$ & p-value \\
\hline $\begin{array}{l}\text { T0 } \\
\text { Mean } \pm \text { SD } \\
\text { Range }\end{array}$ & $\begin{array}{c}96.33 \pm 6.51 \\
90-110\end{array}$ & $\begin{array}{l}94.83 \pm 6.77 \\
80-110\end{array}$ & $>0.05$ \\
\hline $\begin{array}{l}\text { T1 } \\
\text { Mean } \pm \text { SD } \\
\text { Range }\end{array}$ & $\begin{array}{c}95.60 \pm 4.12 \\
90-102 \\
\end{array}$ & $\begin{array}{c}82.60 \pm 6.20 \\
64-88\end{array}$ & $<0.001 * *$ \\
\hline $\begin{array}{l}\text { T2 } \\
\text { Mean } \pm \text { SD } \\
\text { Range }\end{array}$ & $\begin{array}{c}92.73 \pm 7.14 \\
82-107\end{array}$ & $\begin{array}{c}77.45 \pm 6.80 \\
65-90\end{array}$ & $<0.001 * *$ \\
\hline $\begin{array}{l}\text { T3 } \\
\text { Mean } \pm \text { SD } \\
\text { Range }\end{array}$ & $\begin{array}{c}90.28 \pm 7.07 \\
83-103\end{array}$ & $\begin{array}{c}77.73 \pm 8.38 \\
65-91\end{array}$ & $<0.001 * *$ \\
\hline $\begin{array}{l}\text { T4 } \\
\text { Mean } \pm \text { SD } \\
\text { Range }\end{array}$ & $\begin{array}{l}89.58 \pm 4.92 \\
80-97\end{array}$ & $\begin{array}{l}88.40 \pm 6.00 \\
73-97\end{array}$ & $>0.05$ \\
\hline $\begin{array}{l}\text { T5 } \\
\text { Mean } \pm \text { SD } \\
\text { Range }\end{array}$ & $\begin{array}{c}88.73 \pm 5.27 \\
80-96 \\
\end{array}$ & $\begin{array}{c}87.45 \pm 5.95 \\
80-100 \\
\end{array}$ & $>0.05$ \\
\hline $\begin{array}{l}\text { T6 } \\
\text { Mean } \pm \text { SD } \\
\text { Range }\end{array}$ & $\begin{array}{c}87.45 \pm 5.62 \\
80-100\end{array}$ & $\begin{array}{c}88.73 \pm 6.90 \\
80-103\end{array}$ & $>0.05$ \\
\hline $\begin{array}{l}\text { T7 } \\
\text { Mean } \pm \text { SD } \\
\text { Range }\end{array}$ & $\begin{array}{c}89.15 \pm 6.95 \\
80-100\end{array}$ & $\begin{array}{l}89.20 \pm 4.98 \\
80-98\end{array}$ & $>0.05$ \\
\hline $\begin{array}{l}\text { T8 } \\
\text { Mean } \pm \text { SD } \\
\text { Range }\end{array}$ & $\begin{array}{c}86.68 \pm 6.18 \\
78-103\end{array}$ & $\begin{array}{l}86.98 \pm 5.25 \\
78-94\end{array}$ & $>0.05$ \\
\hline $\begin{array}{l}\text { T9 } \\
\text { Mean } \pm \text { SD } \\
\text { Range }\end{array}$ & $\begin{array}{l}89.75 \pm 0.98 \\
88-91\end{array}$ & $\begin{array}{l}87.71 \pm 3.28 \\
85-91\end{array}$ & $>0.05$ \\
\hline $\begin{array}{l}\text { T10 } \\
\text { Mean } \pm \text { SD } \\
\text { Range }\end{array}$ & $\begin{array}{l}90.75 \pm 5.14 \\
86-96\end{array}$ & $\begin{array}{l}87.22 \pm 3.68 \\
83-91\end{array}$ & $>0.05$ \\
\hline $\begin{array}{l}\text { T11 } \\
\text { Mean } \pm \text { SD } \\
\text { Range }\end{array}$ & $\begin{array}{c}86.55 \pm 9.37 \\
77-96 \\
\end{array}$ & $\begin{array}{l}85.71 \pm 6.32 \\
80-93\end{array}$ & $>0.05$ \\
\hline $\begin{array}{l}\text { T12 } \\
\text { Mean } \pm \text { SD } \\
\text { Range }\end{array}$ & $\begin{array}{c}88.74 \pm 7.34 \\
81-96 \\
\end{array}$ & $\begin{array}{c}87.36 \pm 9.93 \\
85-98\end{array}$ & $>0.05$ \\
\hline $\begin{array}{l}\text { T13 } \\
\text { Mean } \pm \text { SD } \\
\text { Range }\end{array}$ & $\begin{array}{c}86.19 \pm 9.62 \\
76-97\end{array}$ & $\begin{array}{c}85.22 \pm 7.36 \\
77-94\end{array}$ & $>0.05$ \\
\hline $\begin{array}{l}\text { T14 } \\
\text { Mean } \pm \text { SD } \\
\text { Range }\end{array}$ & $\begin{array}{c}86.62 \pm 3.21 \\
84-91\end{array}$ & $\begin{array}{c}84.91 \pm 6.69 \\
78-92\end{array}$ & $>0.05$ \\
\hline
\end{tabular}


The mean blood pressure (MBP) in corresponding times shows no statistically

significant difference between the two Table (4): Comparison between control group and midazolam group according to mean arterial blood pressure

\begin{tabular}{|c|c|c|c|}
\hline $\begin{array}{l}\text { Mean arterial blood } \\
\text { pressure }(\mathrm{mmHg})\end{array}$ & $\begin{array}{l}\text { Control group } \\
\qquad(n=40)\end{array}$ & $\begin{array}{c}\text { Midazolam group } \\
\qquad(n=40)\end{array}$ & p-value \\
\hline $\begin{array}{l}\text { T0 } \\
\text { Mean } \pm \text { SD } \\
\text { Range } \\
\end{array}$ & $\begin{array}{c}93.20 \pm 4.78 \\
87-98 \\
\end{array}$ & $\begin{array}{c}94.37 \pm 5.14 \\
86-99 \\
\end{array}$ & $>0.05$ \\
\hline $\begin{array}{l}\text { T1 } \\
\text { Mean } \pm \text { SD } \\
\text { Range }\end{array}$ & $\begin{array}{c}91.46 \pm 2.98 \\
88-94\end{array}$ & $\begin{array}{c}92.61 \pm 5.14 \\
88-98\end{array}$ & $>0.05$ \\
\hline $\begin{array}{l}\text { T2 } \\
\text { Mean } \pm \text { SD } \\
\text { Range }\end{array}$ & $\begin{array}{c}80.38 \pm 5.95 \\
74-97\end{array}$ & $\begin{array}{c}80.83 \pm 7.80 \\
70-94\end{array}$ & $>0.05$ \\
\hline $\begin{array}{l}\text { T3 } \\
\text { Mean } \pm \text { SD } \\
\text { Range } \\
\end{array}$ & $\begin{array}{c}83.73 \pm 3.16 \\
77-88 \\
\end{array}$ & $\begin{array}{c}82.85 \pm 3.47 \\
75-88 \\
\end{array}$ & $>0.05$ \\
\hline $\begin{array}{l}\text { T4 } \\
\text { Mean } \pm \text { SD } \\
\text { Range } \\
\end{array}$ & $\begin{array}{c}83.10 \pm 3.44 \\
79-88 \\
\end{array}$ & $\begin{array}{c}82.03 \pm 4.42 \\
73-88 \\
\end{array}$ & $>0.05$ \\
\hline $\begin{array}{l}\text { T5 } \\
\text { Mean } \pm \text { SD } \\
\text { Range }\end{array}$ & $\begin{array}{c}86.55 \pm 3.86 \\
81-92 \\
\end{array}$ & $\begin{array}{c}85.83 \pm 4.27 \\
78-92 \\
\end{array}$ & $>0.05$ \\
\hline $\begin{array}{l}\text { T6 } \\
\text { Mean } \pm \text { SD } \\
\text { Range } \\
\end{array}$ & $\begin{array}{c}86.25 \pm 5.37 \\
79-94\end{array}$ & $\begin{array}{c}86.18 \pm 3.64 \\
79-90\end{array}$ & $>0.05$ \\
\hline $\begin{array}{l}\text { T7 } \\
\text { Mean } \pm \text { SD } \\
\text { Range }\end{array}$ & $\begin{array}{c}84.38 \pm 5.07 \\
77-94 \\
\end{array}$ & $\begin{array}{c}84.43 \pm 2.44 \\
79-90 \\
\end{array}$ & $>0.05$ \\
\hline $\begin{array}{l}\text { T8 } \\
\text { Mean } \pm \text { SD } \\
\text { Range }\end{array}$ & $\begin{array}{c}87.63 \pm 4.67 \\
80-94\end{array}$ & $\begin{array}{c}85.00 \pm 5.08 \\
75-92\end{array}$ & $>0.05$ \\
\hline $\begin{array}{l}\text { T9 } \\
\text { Mean } \pm \text { SD } \\
\text { Range }\end{array}$ & $\begin{array}{c}88.00 \pm 4.96 \\
82-95 \\
\end{array}$ & $\begin{array}{c}87.60 \pm 4.98 \\
80-97 \\
\end{array}$ & $>0.05$ \\
\hline $\begin{array}{l}\text { T10 } \\
\text { Mean } \pm \text { SD } \\
\text { Range } \\
\end{array}$ & $\begin{array}{c}89.13 \pm 5.40 \\
81-98 \\
\end{array}$ & $\begin{array}{c}87.90 \pm 5.00 \\
79-96 \\
\end{array}$ & $>0.05$ \\
\hline $\begin{array}{l}\text { T11 } \\
\text { Mean } \pm \text { SD } \\
\text { Range }\end{array}$ & $\begin{array}{c}81.35 \pm 8.44 \\
74-90\end{array}$ & $\begin{array}{c}83.35 \pm 7.67 \\
77-92\end{array}$ & $>0.05$ \\
\hline $\begin{array}{l}\text { T12 } \\
\text { Mean } \pm \text { SD } \\
\text { Range } \\
\end{array}$ & $\begin{array}{c}81.23 \pm 8.99 \\
72-90 \\
\end{array}$ & $\begin{array}{c}79.98 \pm 5.95 \\
73-87 \\
\end{array}$ & $>0.05$ \\
\hline $\begin{array}{l}\text { T13 } \\
\text { Mean } \pm \text { SD } \\
\text { Range } \\
\end{array}$ & $\begin{array}{c}81.0 \pm 7.5 \\
73-89 \\
\end{array}$ & $\begin{array}{c}82.3 \pm 9.0 \\
74-92 \\
\end{array}$ & $>0.05$ \\
\hline $\begin{array}{l}\text { T14 } \\
\text { Mean } \pm \text { SD } \\
\text { Range }\end{array}$ & $\begin{array}{c}83.0 \pm 10.0 \\
73-90 \\
\end{array}$ & $\begin{array}{c}79.0 \pm 8.0 \\
72-88 \\
\end{array}$ & $>0.05$ \\
\hline
\end{tabular}


APGAR scores were recorded at one and five minutes after birth. There was no statistically significant difference between control group and midazolam group (Table 5).

Table (5): Comparison between control group and midazolam group according to Apgar score of neonate

\begin{tabular}{|c|c|c|c|}
\hline APGAR Score $\quad$ Groups & $\begin{array}{c}\text { Control group } \\
(n=40)\end{array}$ & $\begin{array}{c}\text { Midazolam group } \\
(n=40)\end{array}$ & p-value \\
\hline $1 \mathrm{~min}$ & & & \\
\hline Mean \pm SD & $7.15 \pm 0.64$ & $6.23 \pm 0.73$ & $>0.05$ \\
\hline Range & $6-9$ & $5-7$ & \\
\hline $5 \min$ & & & \\
\hline Mean \pm SD & $8.50 \pm 0.51$ & $8.48 \pm 0.51$ & $>0.05$ \\
\hline Range & $8-9$ & $8-9$ & \\
\hline
\end{tabular}

All delivered neonates were assessed with Neurologic Adaptive Capacity Score $(\mathrm{NACS})$ (value $=0-40)$ at 15 min post- delivery. NACS showed no statistical significant difference between groups (Table 6).

Table (6): Comparison between control group midazolam group according to NACS at 15 min. of neonate

\begin{tabular}{|l|c|c|c|}
\hline NACS at 15 min $\quad$ Groups & $\begin{array}{c}\text { Control group } \\
(\boldsymbol{n}=\mathbf{4 0})\end{array}$ & $\begin{array}{c}\text { Midazolam group } \\
(\boldsymbol{n}=\mathbf{4 0})\end{array}$ & p-value \\
\hline Mean \pm SD & $34.75 \pm 1.78$ & $32.80 \pm 1.42$ & \multirow{2}{*}{$>0.05$} \\
\cline { 1 - 2 } Range & $32-37$ & $31-36$ & \\
\hline
\end{tabular}

Umbilical Cord Artery Blood Gases (UABG) were collected just after delivery. Data obtained showed no statistically

significant differences between groups (Table 7).

Table (7): Comparison between control group midazolam group according to umbilical ABG of neonate

\begin{tabular}{|c|c|c|c|}
\hline Umbilical ABG $\quad$ Groups & $\begin{array}{c}\text { Control group } \\
(n=40)\end{array}$ & $\begin{array}{c}\text { Midazolam group } \\
(n=40)\end{array}$ & p-value \\
\hline $\begin{array}{l}\mathbf{p H} \\
\text { Mean } \pm \text { SD } \\
\text { Range }\end{array}$ & $\begin{array}{c}7.29 \pm 0.01 \\
7.26-7.3 \\
\end{array}$ & $\begin{array}{c}7.25 \pm 0.02 \\
7.22-7.3\end{array}$ & $>0.05$ \\
\hline $\begin{array}{l}\text { Pa Co2 } \\
\text { Mean } \pm \text { SD } \\
\text { Range }\end{array}$ & $\begin{array}{c}50.48 \pm 3.38 \\
44-55 \\
\end{array}$ & $\begin{array}{c}51.33 \pm 4.60 \\
46-57\end{array}$ & $>0.05$ \\
\hline $\begin{array}{l}\text { Pa O2 } \\
\text { Mean } \pm \text { SD } \\
\text { Range }\end{array}$ & $\begin{array}{c}21.73 \pm 2.75 \\
18-27 \\
\end{array}$ & $\begin{array}{c}21.40 \pm 1.28 \\
20-24\end{array}$ & $>0.05$ \\
\hline $\begin{array}{l}\text { Hco3 } \\
\text { Mean } \pm \text { SD } \\
\text { Range }\end{array}$ & $\begin{array}{c}22.88 \pm 1.47 \\
20-25 \\
\end{array}$ & $\begin{array}{c}22.03 \pm 1.75 \\
19-24 \\
\end{array}$ & $>0.05$ \\
\hline $\begin{array}{l}\text { Base deficit } \\
\text { Mean } \pm \text { SD } \\
\text { Range }\end{array}$ & $\begin{array}{c}-4.21 \pm 0.38 \\
-4.7-3.6 \\
\end{array}$ & $\begin{array}{c}-4.12 \pm 0.47 \\
-4.7-3 \\
\end{array}$ & $>0.05$ \\
\hline
\end{tabular}




\section{DISCUSSION}

The current study was designed as a prospective placebo controlled double blinded clinical study. It was conducted on 80 patients presented for elective cesarean section at full term pregnancy. It was designed to investigate the possibility that midazolam could be used for sedation during cesarean section with spinal anesthesia, for effective control of parturient anxiety, while not compromising neonatal safety.

This study has shown a significant improvement of preoperative anxiety in all parturients scheduled for elective cesarean section under spinal anesthesia who received intravenous midazolam in dose of $0.035 \mathrm{mg} / \mathrm{kg}$ as measured by APAIS score over time, beside significant decrease in their heart rate during operation. There was no significant difference between the two groups regarding maternal blood pressure throughout the procedure.

Regarding neonates APGAR score showed no statistically significant differences between the two groups at 1 $\min \& 5$ min.

NACS score and Umbilical cord artery blood gases showed no statistically significant differences between the two groups.

Premedication started $30 \mathrm{~min}$ before the induction of spinal anesthesia to allow enough time for suppression of anxiety. This was in contrast to Fr?lich et al. (2006) who gave their premedication immediately before the spinal anesthesia procedure (Mokhtar et al., 2016).

Dosage is also an important factor to be considered, we chose the midazolam dose $\quad(0.035 \quad \mathrm{mg} / \mathrm{kg})$ based on recommendations of previous studies (Senel and Mergan 2014 and Mokhtar et al., 2016).

We chose a single bolus based on body weight; however, in clinical practice, some may prefer to titrate iv drugs to effect. Thus, our results cannot be compared to other studies with repeated doses or iv infusion.

Fr?lich et al. (2006) used a single iv dose of $0.02 \mathrm{mg} / \mathrm{kg}$ midazolam plus 1 $\mu \mathrm{g} / \mathrm{kg}$ fentanyl to parturients undergoing cesarean delivery shortly before spinal needle insertion. Similar to our study, Apgar scores showed no difference between the treated group and the control group (Mokhtar et al., 2016).

In contrast, Senel and Mergan (2014) gave a single iv dose of $0.025 \mathrm{mg} / \mathrm{kg}$ midazolam to parturients $30 \mathrm{~min}$ before cesarean delivery. They evaluated maternal anxiety using APAIS score and newborns with Apgar and NACS scores. They reported significantly lower anxiety scores in the treated groups, without any adverse neonatal outcome. However, they recommended further clinical trials with higher sample size to assess the larger doses of midazolam.

The limitations of this study may be due to the high selectivity of patients and sample size.

\section{CONCLUSION}

Preoperative sedation at $30 \mathrm{mins}$ pre anesthetic induction was found to be associated with improved preoperative anxiety, postoperative satisfaction, and maternal hemodynamics in parturients scheduled for elective cesarean delivery, 
with no adverse effects on the neonates especially at dose of $0.035 \mathrm{mg} / \mathrm{kg}$.

\section{REFERENCES}

1. Amin, S. M., Amr, Y. M., Fathy, S. M. and Alzeftawy, A. E. (2011): Maternal and neonatal effects of nalbuphine given immediately before induction of general anesthesia for elective cesarean section. Saudi journal of anaesthesia, 5(4): 371-375.

2. Carroll JK, Cullinan E, Clarke $L$ and Davis NF (2012): The role of anxiolytic premedication in reducing preoperative anxiety. British Journal of Nursing, 21(8):479-483.

3. Goebel, S., Kaup, L. and Mehdorn, H. M. (2011): Measuring preoperative anxiety in patients with intracranial tumors: the Amsterdam preoperative anxiety and information scale. Journal of neurosurgical anesthesiology, 23(4), 297-303.

4. Griffin C, Kaye A, Bueno $\mathbf{F}$ and Kaye $A$ (2013): Benzodiazepine Pharmacology and Central Nervous System-Mediated Effects. The Ochsner Journal, 13(2):214-23.

5. Hashemi SJ, Jabalameli M and Mokhtary F (2015): Effects of different anesthetic techniques on neurologic and adaptation capacity in newborn with elective cesarean section. Adv Biomed Res., 4:249-254.

6. Kemper AR, Mahle WT and Martin GR (2011): Strategies for implementing screening for critical congenital heart disease. Pediatrics, 128:5, 1-9.
7. Martin JA, Hamilton BE, Sutton PD, Ventura SJ, Mathews TJ, Kirmeyer S and Osterman MJ. (2010): Births: final data for 2007. Natl Vital Stat Rep., 58:1-85.

8. Mokhtar, A. M., Elsakka, A. I. and Ali, H. M. (2016): Premedication with midazolam prior to cesarean delivery in preeclamptic parturients: A randomized controlled trial. Anesthesia, essays and researches, 10(3): 631-636.

9. Patki A and Shelgaonkar VC (2011): A comparison of equisedative infusions of propofol and midazolam for conscious sedation during spinal anesthesia - a prospective randomized study. J Anaesthesiol Clin Pharmacol., 27:47-53.

10. Sakata K, Yoshimura N, Tanabe K, Kito $K$, Nagase $K$ and Iida $H$ (2017): Prediction of hypotension durin spinal anesthesia for elective cesarean section by altered heart rate variability induced by postural change. Int $\mathbf{J}$ Obstet Anesth., 29: 34-8.

11. Senel AC and Mergan F (2014): Premedication with midazolam prior to caesarean section has no neonatal adverse effects. Braz J Anesthesiol., 64:16-21.

12. Zhou C, Zhu Y, Bao Z, Wang $X$ and Liu $Q$ (2018): Efficacy of ondansetron for spinal anesthesia during cesarean section: A metaanalysis of randomized trials. J Int Med Res., 46 (2):654-62. 


\section{تأثثير عقار الميدازو لام قبل الولادة القيصرية الاختيارية على

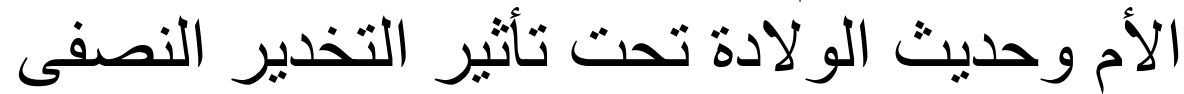

عماد عبد الحميد شعبان ـ محمد عبد الجواد عبد الحليم أبو السعود ـ محمود عماد

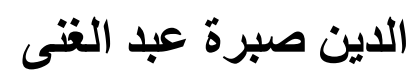

$$
\text { قسم التخدير والرعاية المركزة ـ كلية طب الأزهر }
$$



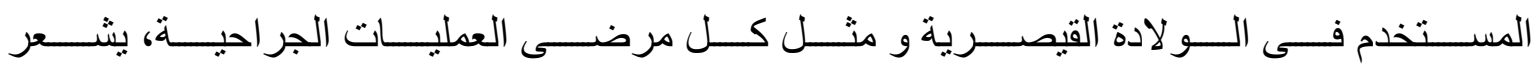

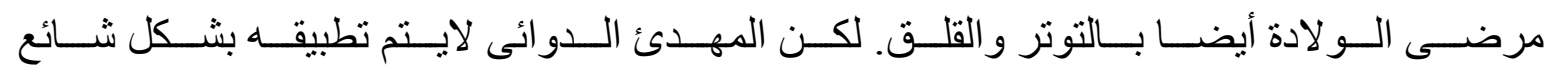

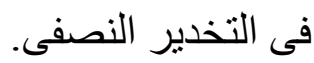

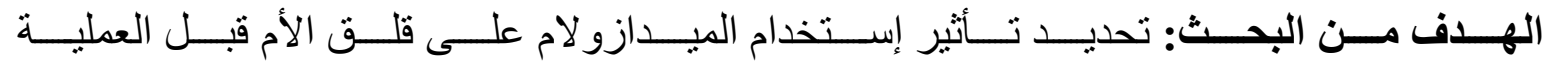

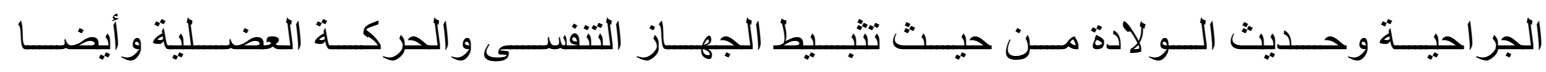

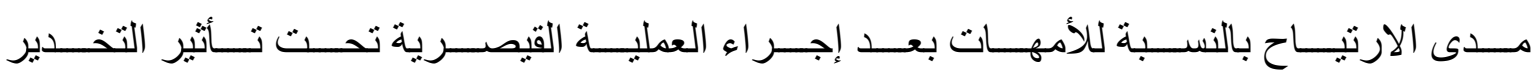
النصفى.

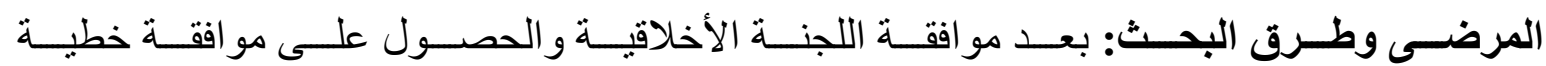

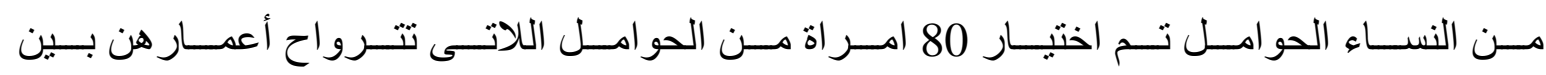

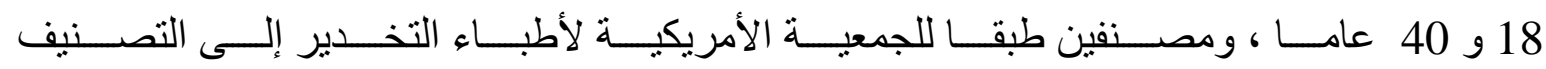

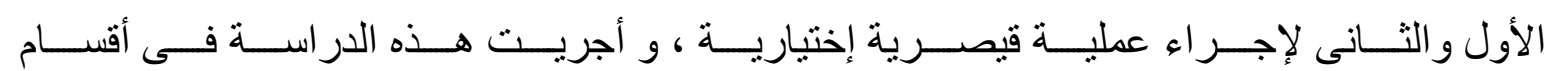

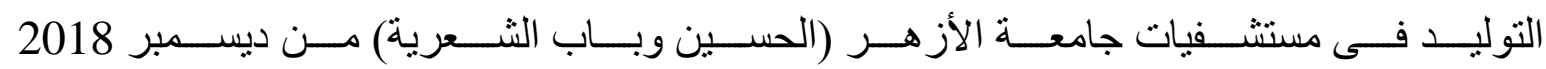
حتى يونيو 2019.

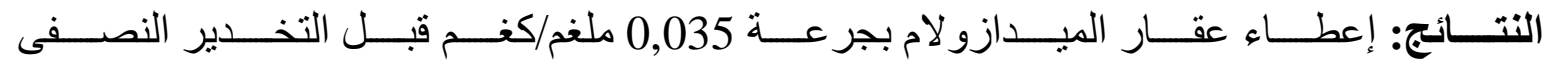


تأثير سلبى على استقرار الدورة الدموية و حديثى الولادة.

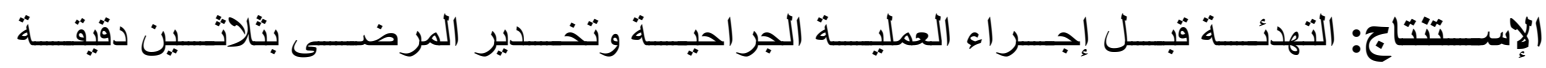

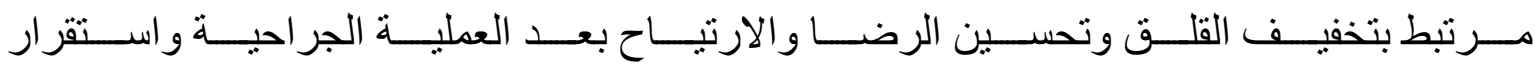
الدورة الدموية بالنسبة للأمهات الخاضعات للو لادة القيصرية. 